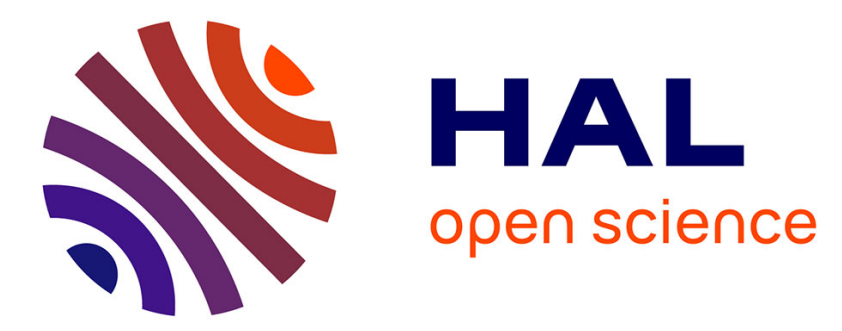

\title{
Wechselwirkung zwischen der Anisotropie der Schwind- und Quellmaße sowie Holzfeuchte-Änderungen in der Ebene von gekrümmtem Brettschichtholz
}

\author{
Matthias Frese
}

\section{- To cite this version:}

Matthias Frese. Wechselwirkung zwischen der Anisotropie der Schwind- und Quellmaße sowie Holzfeuchte-Änderungen in der Ebene von gekrümmtem Brettschichtholz. European Journal of Wood and Wood Products, 2010, 69 (3), pp.359-367. 10.1007/s00107-010-0443-9 . hal-00615325

\section{HAL Id: hal-00615325 \\ https://hal.science/hal-00615325}

Submitted on 19 Aug 2011

HAL is a multi-disciplinary open access archive for the deposit and dissemination of scientific research documents, whether they are published or not. The documents may come from teaching and research institutions in France or abroad, or from public or private research centers.
L'archive ouverte pluridisciplinaire HAL, est destinée au dépôt et à la diffusion de documents scientifiques de niveau recherche, publiés ou non, émanant des établissements d'enseignement et de recherche français ou étrangers, des laboratoires publics ou privés. 


\title{
Wechselwirkung zwischen der Anisotropie der Schwind- und Quellmaße sowie Holzfeuchte-Änderungen in der Ebene von gekrümmtem Brettschichtholz
}

\author{
Matthias Frese \\ Universität Karlsruhe, Lehrstuhl für Ingenieurholzbau und Baukonstruktionen, \\ Kaiserstraße 12, 76131 Karlsruhe, Germany \\ Matthias.Frese@holz.uka.de
}

\begin{abstract}
Zusammenfassung Erkenntnisse zur Anisotropie der Schwind- und Quellmaße in der polarorthotrop aufgebauten Hirnfläche von Nadelholz werden auf die Ebene von gekrümmtem Brettschichtholz aus Nadelholz übertragen. Die Anisotropie bei Brettschichtholz ist dabei aus ingenieurtechnischer Sicht durch das Verhältnis von Schwind- und Quellmaß in Faserrichtung zum Maß quer zur Faser von 1/24 gekennzeichnet. Bei Vollholzquerschnitten liegt das Verhältnis tangential/radial in der Regel bei 2/1. Mit einfachen Modellbetrachtungen an Kreisringstücken aus Brettschichtholz wird gezeigt, dass die ausgeprägte Anisotropie bei gekrümmtem Brettschichtholz und Holzfeuchte-Änderungen im hygroskopischen Bereich Krümmungsänderungen gemeinsam bedingen. Das kann bei Tragwerken Zwangs- und Eigenspannungen zur Folge haben.
\end{abstract}

\section{Interaction between differential shrinkage and moisture content variations in the plane of curved glulam}

\footnotetext{
Abstract Findings on the differential shrinkage in the cross-section of solid timber as cylindrically orthotropic material are applied to the plane of curved softwood glulam. From an engineering point of view, the differential shrinkage in the plane of curved glulam is characterised by a ratio of longitudinal shrinkage to averaged shrinkage perpendicular to the grain of 1:24. For solid timber, the ratio tangential to radial is about 2:1. By means of modelling glulam as part of a circular ring it is shown that curvature variations are caused by both pronounced differential shrinkage in the plane of curved glulam and moisture content variations in the hygroscopic range of wood. This can result in constraining and internal stresses in structural systems.
} 


\section{Einleitung}

Die Anisotropie der Schwind- und Quellmaße (im Folgenden kurz: Anisotropie) in der Hirnfläche bzw. im Querschnitt eines Baumstammes ist eine wichtige technologische Holzeigenschaft (vgl. DIN 52184:1979). Sie erfordert bei der Holzverarbeitung seit jeher sorgfältige Beachtung, weil Änderungen der Holzfeuchte, auch im bereits getrockneten Holz, aufgrund der Änderung des Umgebungsklimas während der Herstellung und der Nutzung von Holz und Holzprodukten kaum zu vermeiden sind. Bei etlichen Nutzhölzern finden sich in der polar-orthotrop aufgebauten Hirnfläche ausgeprägte Unterschiede zwischen der differentiellen Quellung in tangentialer $\left(q_{\mathrm{t}}\right)$ und in radialer Richtung $\left(q_{\mathrm{r}}\right)$, wobei die Anistropie in der Hirnfläche $\left(A_{\mathrm{q}}\right)$ bei einheimischen Bauhölzern (z. B. Fichte, Kiefer, Lärche, Tanne, Buche und Eiche) in der Regel 2 beträgt, s.

Gleichung (1)(1). Das Verhältnis gilt ebenso für die Schwindmaße in tangentialer $\left(\beta_{\mathrm{t}}\right)$ und in radialer Richtung $\left(\beta_{\mathrm{r}}\right)$. Diese Erkenntnisse gehen unter anderem auf grundlegende Betrachtungen und Untersuchungen von Keylwerth (1951 und 1962) zurück.

$$
A_{\mathrm{q}}=\frac{q_{\mathrm{t}}}{q_{\mathrm{r}}} \approx 2
$$

Da das tangentiale und das radiale Schwind- und Quellmaß in den zwei zueinander orthogonalen anatomischen Hauptrichtungen, parallel und senkrecht zu den Jahrringen, wirken, werden Holzquerschnitte um so mehr formtreu schwinden (für Quellen gilt das sinngemäß), je besser ihre Kanten den beiden Hauptrichtungen folgen (Keylwerth 1951); umgekehrt gilt, dass sich Querschnittskanten beim Schwinden um so mehr krümmen und verzerren, je mehr sie von den Hauptrichtungen abweichen (Kollmann 1981). In diesem Sinne gab Keylwerth (1944/1945) für Stammscheibenausschnitte bereits eine Formel (2)(2) zur Berechnung des Öffnungswinkels für Schwinden vom nassen zum darrtrockenen Zustand an (vgl. Bild 1Bild 1).

$$
\varphi_{0}=\varphi_{\mathrm{w}} \cdot \frac{1-\beta_{\mathrm{t}}}{1-\beta_{\mathrm{r}}}
$$

Darin sind $\varphi_{0}$ und $\varphi_{\mathrm{w}}$ die Öffnungswinkel im darrtrockenen bzw. nassen Zustand. Er berichtet, dass Stücke, die das Mark beinhalten zum Reißen neigen, wenn die 
Winkelschwindung größer oder gleich ist als die Bruchdehnung des Holzes in tangentialer Richtung.

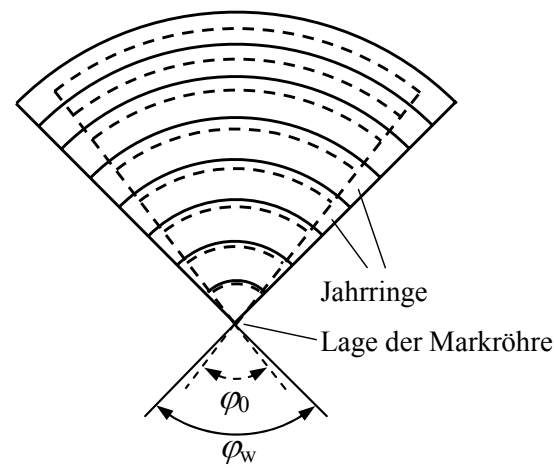

Bild 1 Schwinden beim Stammscheibenausschnitt; Zustand nach dem Schwinden (gestrichelte Linien), unmaßstäblich

Fig. 1 Schematic differential shrinkage of a tree disk sector; after shrinkage (dashed lines), not scaled

KublerKubler (1975) führte Trocknungsexperimente an Baumscheiben unterschiedlicher nordamerikanischer Holzarten durch und beobachtete, der Theorie des anisotropen Schwindens entsprechend, bei Holzfeuchten unter etwa $15 \%$ radial in den Baumscheiben verlaufende Risse. HsuHst und Tang (1975) zeigten mit einer analytischen Lösung, dass bei konstanter Feuchteabgabe und bei idealen polar-orthotropen Materialeigenschaften markfreie Brettquerschnitte ohne die Entstehung von Eigenspannungen schwinden. Zu den Ursachen der für Baumscheiben typischen V-förmigen Risse als Folge des Schwindens gibt es im Schrifttum weitere Arbeiten, z.B. von TauchertFauchert und Hsu (1977) sowie KangKang und Lee (2002). In diesen Arbeiten werden Einflüsse aus nicht konstanten Feuchteverhältnissen auf Spannungszustände in der Hirnfläche theoretisch untersucht. KangKang und Lee (2004) bestätigen in ihrer experimentellen Arbeit an Lärchenholz bisherige Erkenntnisse: Ihre Versuche zeigen u. a., dass in vollständigen Baumscheiben, bei denen das freie Schwinden behindert ist, Eigenspannungen und bei sehr ausgeprägter Anisotropie gehäuft VRisse entstehen. Unter der Annahme, dass markfreie Stammscheibenausschnitte eigenspannungsfrei schwinden, führten sie Versuche zur Ermittlung der radialen und tangentialen Schwindmaße für freies Schwinden durch.

Formatted: Complex Script Font: 12 pt, German Germany 
Zwischen der polar-orthotrop aufgebauten Hirnfläche und der Ebene von künstlich gekrümmtem Brettschichtholz besteht eine umgekehrte strukturelle Analogie: Während in der Hirnfläche die Anisotropie durch das Verhältnis 2 gekennzeichnet ist, liegt in der Ebene von gekrümmtem Brettschichtholz eine gegensätzliche und wesentlich stärker ausgeprägte Anisotropie $\left(A_{\alpha, \mathrm{BSH}}\right)$ vor, deren Grad für Brettschichtholz mit Gleichung (3)(3) abgeschätzt werden kann.

$$
A_{\alpha, \mathrm{BSH}}=\frac{\alpha_{\ell}}{\alpha_{\mathrm{t} / \mathrm{r}}} \approx \frac{0,01}{0,24}=\frac{1}{24}
$$

Darin sind $\alpha_{\ell}$ das Schwind- und Quellmaß in Faserrichtung bzw. in globaler tangentialer Richtung und $\alpha_{t / r}$ ein gewichtetes quer zur Faser bzw. in globaler radialer Richtung. Die Zahlenwerte in Gleichung (3)(3) entsprechen den ingenieurtechnischen Rechenwerten (in Anlehnung an internationale Veröffentlichungen im Folgenden mit $\alpha$ bezeichnet) für Nadelholz (DIN 1052:2008) und geben jeweils die prozentuale Längenänderung je Prozent Holzfeuchte-Änderung im hygroskopischen Bereich an. Wenn eine Anisotropie von 2 in der Hirnfläche bekanntermaßen sorgfältige Beachtung erfordert, so sollte der künstlich geschaffenen und daher spezifischen Anisotropie in der Ebene von gekrümmtem Brettschichtholz mit einem Verhältnis von etwa 1/24 eine mindestens ebenso sorgfältige Beachtung zukommen. Dennoch finden sich im Schrifttum über das von der Holzfeuchtigkeit abhängige Verformungsverhalten von gekrümmten Brettschichtholz-Bereichen wie offenen Kreisringstücken, z.B. Teilbereiche in Satteldachträgen, und über entsprechende Spannungszustände nur wenige Hinweise. Larsentarsen und Riberholt (1983) geben Berechnungsansätze an für die von der Holzfeuchte-Änderung abhängige Verformung (hinsichtlich der pt, German Germany vertikalen Verschiebung des Firstpunktes) von Dreigelenkrahmen aus Brettschichtholz mit gekrümmten oder keilgezinkten Rahmenecken. Hoffmeyer (1995) berichtet darüber in Auszügen. In ihren Ansätzen ist die Verformung des Rahmens von der durch die Holzfeuchte-Änderung bedingten Winkeländerung in der gekrümmten oder keilgezinkten Rahmenecke abhängig. Es kann angenommen werden, dass bei der Planung und Bemessung allgemein von gekrümmten Brettschichtholz-Bauteilen nur selten entsprechenden Berechnungen durchgeführt werden. Dieser Beitrag widmet sich daher grundsätzlichen ingenieurmäßigen Überlegungen zum Verformungsverhalten in der Ebene von gekrümmtem Brettschichtholz aufgrund der Wechselwirkung zwischen der für Brettschichtholz 
spezifischen Anisotropie und Holzfeuchte-Änderungen im hygroskopischen

Bereich mit $5 \%<u<20 \%$.

\section{Motivation}

Die Beweggründe für Überlegungen zu dieser Wechselwirkung liegen in der bis heute beobachteten Querzugproblematik bei Satteldachträgern aus

Brettschichtholz (vor allem mit geneigtem Untergurt). Dieses Problem ist i. d. R. durch im gekrümmten Bereich verlaufende offene Risse in Faserrichtung, teilweise auch im Holzbereich nahe der Klebefuge, gekennzeichnet. Nach heutigen Erkenntnissen wirken hinsichtlich der Beanspruchung durch Querzugspannungen in gekrümmten Bereichen solche Spannungen, die aus äußeren Lasten und aus Klimawechseln, mit der Folge eines Feuchtegefälles in Dickenrichtung, herrühren (vgl. z. B. DIN 1052:2008 sowie Möhler und Steck 1980).

In der kürzlich abgeschlossenen Forschungsarbeit Blaß und Frese (2010) wurde gezeigt, dass die Querzugproblematik bei Satteldachträgern nicht als grundsätzlich überwunden angesehen werden kann. Es wurden über 170 Querzugschäden an Satteldachträgern in fast ebenso vielen unterschiedlichen Bauwerken mit Standort Bundesgebiet Deutschland analysiert. Dazu wurden die Häufigkeiten der Zusammenhänge zwischen dem Baujahr und dem Zeitraum ab Baujahr bis zur Entdeckung eines Querzugrisses, jeweils in 5-Jahresintervalle | eingeteilt, dargestellt (Tabelle 1Fabelle 1). Demnach wurde man in den späten 1960er Jahren auf die ersten Querzugschäden aufmerksam (eingekreiste 4). 1980 waren es bereits mindestens $4+2+7=13,1985: 4+2+1+7+9+7=30$, 1990: $4+2+1+2+7+9+5+7+7+12=56$ Fälle usw. Die tatsächliche Anzahl dieser spezifischen Querzugschäden ist sehr wahrscheinlich um ein Vielfaches höher, weil bei weitem nicht alle dokumentierten Querzugschäden für die Forschungsarbeit zugänglich waren und weil bei Hallen mit mehreren Binderachsen häufig weitere Träger, manchmal sogar alle Träger, denselben Schaden zeigten. Diese weiteren Fälle sind in Tabelle 1Fabelle 1 nicht gesondert erfasst. 
Grundsätzlich zeigt die Darstellung in Tabelle 1Tabelle 1, dass die

Querzugproblematik trotz entgegenwirkender normativer Anpassungen (DIN

1052:1969, DIN 1052:1988, DIN 1052DIN 1052:2004) und technischer

Verfahren wie eingeklebte Gewindestangen (z.B. Möhler und Hemmer 1981)

langwierig ist: In den 1990er Jahren wurden weiterhin Satteldachträger produziert,

die in den Folgejahren durch Querzugrisse auffällig wurden. Die

Kontingenztabelle zeigt außerdem, dass ein Ende von Querzugschäden bei

Satteldachträgern noch nicht erreicht ist. Bei Bauwerken z. B. aus den 1990er

Jahren sind sehr wahrscheinlich noch Schäden zu erwarten.

Angesichts der Fülle der Querzugschäden, die gewissermaßen immer auch im

Zusammenhang mit Schwinden bzw. Quellen in der Ebene gekrümmter Bereiche standen, entstand die Überlegung, ob umgekehrt analog zur Anisotropie in der

Hirnfläche auch in der Ebene gekrümmter Bereiche von Brettschichtholz

schädliche Querzugspannungen wirken könnten. 
Tabelle 1 Häufigkeiten der Zusammenhänge zwischen Baujahr (Zeilen) und Zeitraum (Spalten) bis zur Entdeckung von klaffenden Querzugrissen in Satteldachträgern, beide

Merkmalsausprägungen in 5-Jahresintervalle zusammengefasst; die eingekreiste 4 kennzeichnet die frühesten Schäden in der Datensammlung

Table 1 Frequency of the relationship between year of erection (rows) and period of time (columns) until detection of cracks in grain direction in pitched cambered beams, both parameters in 5 year intervals; encircled 4 shows earliest failures in the investigated data 
Ejwwp443_source

\begin{tabular}{|c|c|c|c|c|c|c|c|c|c|}
\hline $\begin{array}{l}\text { Frequency } \\
\text { Percent } \\
\text { Row Pct } \\
\text { Col Pct }\end{array}$ & $00-05$ & $06-10$ & $\mid 11-15$ & $16-20$ & $21-25$ & $26-30$ & $31-35$ & $\begin{array}{l}\text { keine } \\
\text { Angabe }\end{array}$ & Total \\
\hline $1900-1965$ & $\begin{array}{r}0 \\
0.00 \\
0.00 \\
0.00\end{array}$ & $\begin{array}{r}0 \\
0.00 \\
0.00 \\
0.00\end{array}$ & $\begin{array}{r}0 \\
0.00 \\
0.00 \\
0.00\end{array}$ & $\begin{array}{r}0 \\
0.00 \\
0.00 \\
0.00\end{array}$ & $\begin{array}{r}0 \\
0.00 \\
0.00 \\
0.00\end{array}$ & $\begin{array}{r}0 \\
0.00 \\
0.00 \\
0.00\end{array}$ & $\begin{array}{r}1 \\
0.58 \\
50.00 \\
20.00\end{array}$ & $\begin{array}{r}1 \\
0.58 \\
50.00 \\
3.33\end{array}$ & $\begin{array}{r}2 \\
1.17\end{array}$ \\
\hline $1966-1970$ & $\begin{array}{r}4 \\
2.34 \\
40.00 \\
8.00\end{array}$ & $\begin{array}{r}2 \\
1.17 \\
20.00 \\
7.14\end{array}$ & $\begin{array}{r}1 \\
0.58 \\
10.00 \\
4.55\end{array}$ & $\begin{array}{r}2 \\
1.17 \\
20.00 \\
11.11\end{array}$ & $\begin{array}{r}0 \\
0.00 \\
0.00 \\
0.00\end{array}$ & $\begin{array}{r}1 \\
0.58 \\
10.00 \\
8.33\end{array}$ & $\begin{array}{r}0 \\
0.00 \\
0.00 \\
0.00\end{array}$ & $\begin{array}{r}0 \\
0.00 \\
0.00 \\
0.00\end{array}$ & $\begin{array}{r}10 \\
5.85\end{array}$ \\
\hline $1971-1975$ & $\begin{array}{r}7 \\
4.09 \\
20.59 \\
14.00\end{array}$ & $\begin{array}{r}9 \\
5.26 \\
26.47 \\
32.14\end{array}$ & $\begin{array}{r}5 \\
2.92 \\
14.71 \\
22.73\end{array}$ & $\begin{array}{r}4 \\
2.34 \\
11.76 \\
22.22\end{array}$ & $\begin{array}{r}3 \\
1.75 \\
8.82 \\
50.00\end{array}$ & $\begin{array}{r}2 \\
1.17 \\
5.88 \\
16.67\end{array}$ & $\begin{array}{r}4 \\
2.34 \\
11.76 \\
80.00\end{array}$ & $\begin{array}{r}0 \\
0.00 \\
0.00 \\
0.00\end{array}$ & $\begin{array}{r}34 \\
19.88\end{array}$ \\
\hline $1976-1980$ & $\begin{array}{r}7 \\
4.09 \\
22.58 \\
14.00\end{array}$ & $\begin{array}{r}7 \\
4.09 \\
22.58 \\
25.00\end{array}$ & $\begin{array}{r}1 \\
0.58 \\
3.23 \\
4.55\end{array}$ & $\begin{array}{r}6 \\
3.51 \\
19.35 \\
33.33\end{array}$ & $\begin{array}{r}1 \\
0.58 \\
3.23 \\
16.67\end{array}$ & $\begin{array}{r}9 \\
5.26 \\
29.03 \\
75.00\end{array}$ & $\begin{array}{r}0 \\
0.00 \\
0.00 \\
0.00\end{array}$ & $\begin{array}{r}0 \\
0.00 \\
0.00 \\
0.00\end{array}$ & $\begin{array}{r}31 \\
18.13\end{array}$ \\
\hline $1981-1985$ & $\begin{array}{r}12 \\
7.02 \\
46.15 \\
24.00\end{array}$ & $\begin{array}{r}7 \\
4.09 \\
26.92 \\
25.00\end{array}$ & $\begin{array}{r}5 \\
2.92 \\
19.23 \\
22.73\end{array}$ & $\begin{array}{r}0 \\
0.00 \\
0.00 \\
0.00\end{array}$ & $\begin{array}{r}2 \\
1.17 \\
7.69 \\
33.33\end{array}$ & $\begin{array}{r}0 \\
0.00 \\
0.00 \\
0.00\end{array}$ & $\begin{array}{r}0 \\
0.00 \\
0.00 \\
0.00\end{array}$ & $\begin{array}{r}0 \\
0.00 \\
0.00 \\
0.00\end{array}$ & $\begin{array}{r}26 \\
15.20\end{array}$ \\
\hline $1986-1990$ & $\begin{array}{r}10 \\
5.85 \\
50.00 \\
20.00\end{array}$ & $\begin{array}{r}2 \\
1.17 \\
10.00 \\
7.14\end{array}$ & $\begin{array}{r}2 \\
1.17 \\
10.00 \\
9.09\end{array}$ & $\begin{array}{r}6 \\
3.51 \\
30.00 \\
33.33\end{array}$ & $\begin{array}{r}0 \\
0.00 \\
0.00 \\
0.00\end{array}$ & $\begin{array}{r}0 \\
0.00 \\
0.00 \\
0.00\end{array}$ & $\begin{array}{r}0 \\
0.00 \\
0.00 \\
0.00\end{array}$ & $\begin{array}{r}0 \\
0.00 \\
0.00 \\
0.00\end{array}$ & $\begin{array}{r}20 \\
11.70\end{array}$ \\
\hline $1991-1995$ & $\begin{array}{r}5 \\
2.92 \\
31.25 \\
10.00\end{array}$ & $\begin{array}{r}1 \\
0.58 \\
6.25 \\
3.57\end{array}$ & $\begin{array}{r}8 \\
4.68 \\
50.00 \\
36.36\end{array}$ & $\begin{array}{r}0 \\
0.00 \\
0.00 \\
0.00\end{array}$ & $\begin{array}{r}0 \\
0.00 \\
0.00 \\
0.00\end{array}$ & $\begin{array}{r}0 \\
0.00 \\
0.00 \\
0.00\end{array}$ & $\begin{array}{r}0 \\
0.00 \\
0.00 \\
0.00\end{array}$ & $\begin{array}{r}2 \\
1.17 \\
12.50 \\
6.67\end{array}$ & $\begin{array}{r}16 \\
9.36\end{array}$ \\
\hline $1996-2000$ & $\begin{array}{r}1 \\
0.58 \\
100.00 \\
2.00\end{array}$ & $\begin{array}{r}0 \\
0.00 \\
0.00 \\
0.00\end{array}$ & $\begin{array}{r}0 \\
0.00 \\
0.00 \\
0.00\end{array}$ & $\begin{array}{r}0 \\
0.00 \\
0.00 \\
0.00\end{array}$ & $\begin{array}{r}0 \\
0.00 \\
0.00 \\
0.00\end{array}$ & $\begin{array}{r}0 \\
0.00 \\
0.00 \\
0.00\end{array}$ & $\begin{array}{r}0 \\
0.00 \\
0.00 \\
0.00\end{array}$ & $\begin{array}{r}0 \\
0.00 \\
0.00 \\
0.00\end{array}$ & $\begin{array}{r}1 \\
0.58\end{array}$ \\
\hline $2001-2005$ & $\begin{array}{r}2 \\
1.17 \\
100.00 \\
4.00\end{array}$ & $\begin{array}{r}0 \\
0.00 \\
0.00 \\
0.00\end{array}$ & $\begin{array}{r}0 \\
0.00 \\
0.00 \\
0.00\end{array}$ & $\begin{array}{r}0 \\
0.00 \\
0.00 \\
0.00\end{array}$ & $\begin{array}{r}0 \\
0.00 \\
0.00 \\
0.00\end{array}$ & $\begin{array}{r}0 \\
0.00 \\
0.00 \\
0.00\end{array}$ & $\begin{array}{r}0 \\
0.00 \\
0.00 \\
0.00\end{array}$ & $\begin{array}{r}0 \\
0.00 \\
0.00 \\
0.00\end{array}$ & $\begin{array}{r}2 \\
1.17\end{array}$ \\
\hline keine Angabe & $\begin{array}{r}2 \\
1.17 \\
6.90 \\
4.00\end{array}$ & $\begin{array}{r}0 \\
0.00 \\
0.00 \\
0.00\end{array}$ & $\begin{array}{r}0 \\
0.00 \\
0.00 \\
0.00\end{array}$ & $\begin{array}{r}0 \\
0.00 \\
0.00 \\
0.00\end{array}$ & $\begin{array}{r}0 \\
0.00 \\
0.00 \\
0.00\end{array}$ & $\begin{array}{r}0 \\
0.00 \\
0.00 \\
0.00\end{array}$ & $\begin{array}{r}0 \\
0.00 \\
0.00 \\
0.00\end{array}$ & $\begin{array}{r}27 \\
15.79 \\
93.10 \\
90.00\end{array}$ & $\begin{array}{r}29 \\
16.96\end{array}$ \\
\hline Total & $\begin{array}{r}50 \\
29.24\end{array}$ & $\begin{array}{r}28 \\
16.37\end{array}$ & $\begin{array}{r}22 \\
12.87\end{array}$ & $\begin{array}{r}18 \\
10.53\end{array}$ & $\begin{array}{r}6 \\
3.51\end{array}$ & $\begin{array}{r}12 \\
7.02\end{array}$ & $\begin{array}{r}5 \\
2.92\end{array}$ & $\begin{array}{r}30 \\
17.54\end{array}$ & $\begin{array}{r}171 \\
100.00\end{array}$ \\
\hline
\end{tabular}




\section{Modellbetrachtungen}

Die Folgen der Wechselwirkung zwischen der Anisotropie in der Ebene von gekrümmten Brettschichtholz-Bauteilen und Holzfeuchte-Änderungen $(\Delta u)$ sollen zunächst durch einen Schwindvorgang am Modell in Bild 2Bild 2 verdeutlicht werden. Es steht für einen 1 fach statisch unbestimmt gelagerten Bogen, ein Kreisringstück mit dem Öffnungswinkel $\varphi_{1}$, der Höhe $h_{1}$ und dem inneren Radius $r_{\mathrm{i}}\left(=h_{1}\right)$ im Ausgangszustand, wobei zunächst angenommen wird, dass sowohl der Schubmodul in Bogenebene als auch das Schwindmaß in Faserrichtung $\left(\alpha_{\ell}\right)$ null seien. Alle weiteren Materialeigenschaften seien konstant. Mit gepunkteten Linien ist in Bild 2Bild 2 die Verformungsfigur dargestellt, die sich beim Schwinden (ohne Feuchtegefälle), symmetrisch um die gekrümmte Schwerelinie, einstellt: Die Höhe $h_{1}$ reduziert sich auf $h_{2}$ und die Hirnflächen des verformten Kreisringstücks schließen aufgrund von Verzerrungen einen größeren Winkel ein als diejenigen des unverformten. Zwischen den neuen Radien und den entsprechenden Teilumfängen der gekrümmten Fasern stellt sich kein konstantes Verhältnis ein.

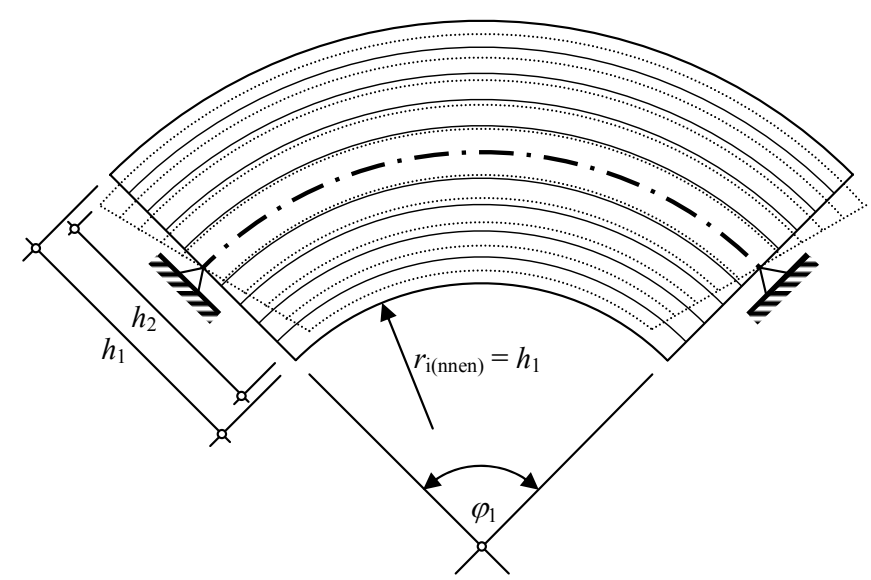

Bild 2 Kreisringstück mit Öffnungswinkel $\varphi_{1}$ (hier beispielhaft $r_{\mathrm{i}}=h_{1}$ ): Beim Schwinden $\left(h_{1} \rightarrow h_{2}\right)$, symmetrisch um die gekrümmte Schwerelinie, kommt es besonders an den Hirnflächen zu Verzerrungen; Zustand nach dem Schwinden (gepunktete Linien) unmaßstäblich

Fig. 2 Part of circular ring with opening angle $\varphi_{1}$ : Under assumption of shear modulus and longitudinal shrinkage equal 0 each shrinkage $\left(h_{1} \rightarrow h_{2}\right)$, symmetrical around the curved cen- 
tre line, causes distortions particularly at the end grain; after shrinkage (dotted lines) not scaled

Da bei Brettschichtholz Gleitungen in Faserrichtung zwischen den gekrümmten Fasern ohne Schädigung der Struktur nicht möglich sind, bedingen die Lagerungsbedingungen und die unterschiedlichen Schwindmaße $\left(\alpha_{\ell}=0, \alpha_{\mathrm{t} / \mathrm{r}}=\right.$ konst.) gemeinsam eine eigentümliche geometrische Unverträglichkeit. Dieser Effekt lässt sich auch mit einer Finite-Elemente-Berechnung, die den obigen Modellannahmen entspricht, darstellen (Bild 3Bild 3). Ein Kreisringstück aus Brettschichtholz, gelagert gemäß Bild 2Bild 2, ist folglich in sich „blockiert“ und eine Holzfeuchte-Abnahme hat schließlich Zwangsspannungen zur Folge.

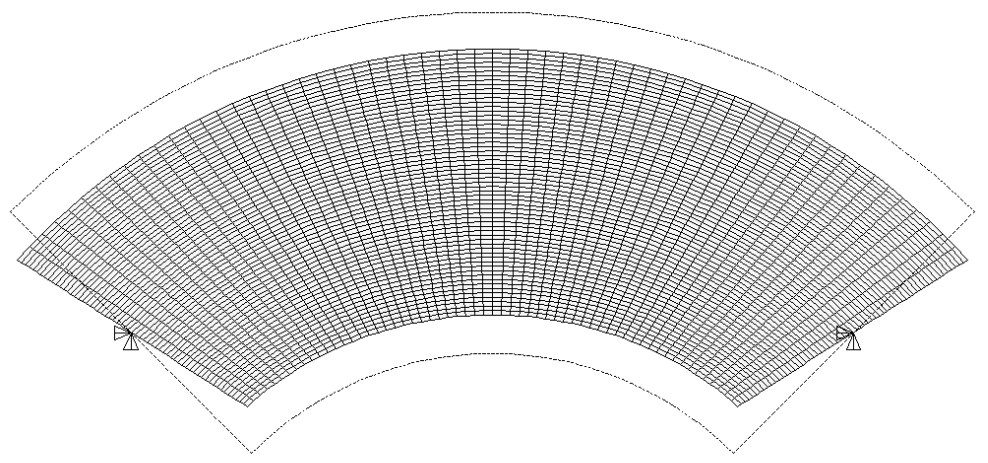

Bild 3 Verformungsfigur (Netz), unmaßstäblich, für das statisch unbestimmt gelagerte Kreisringstück in Bild 2Bild 2

Fig. 3 Deformed structure (mesh), not scaled, part of a circular ring statically indeterminate supported

Wird im Modell bei einem der beiden Auflager, hier beim rechten, die horizontale Verschiebung zugelassen - damit entsteht ein statisch bestimmt gelagertes Kreisringstück - und der Schubmodul wirklichkeitsnah angesetzt, stellt sich aufgrund des nunmehr freien Schwindens die zwängungsfreie Verformungsfigur in Bild 4Bild 4 ein. Es gibt nun weder elastische Dehnungen noch elastische Verzerrungen: Alle vor und nach dem Schwinden einander entsprechenden Teilumfänge sind gleich lang ( $\left.\alpha_{\ell}=0\right)$; die Hirnflächen bilden an den Ecken mit den Tangenten an die Kreisbögen einen rechten Winkel. Während die Krümmung der Schwerelinie beim statisch unbestimmt gelagerten Kreisringstück unverändert bleibt, nimmt sie beim statisch bestimmt gelagerten Kreisringstück zu und die 
Hirnflächen schließen daher einen größeren Winkel ein als vor dem Schwinden. Für Quellen gilt das sinngemäß.

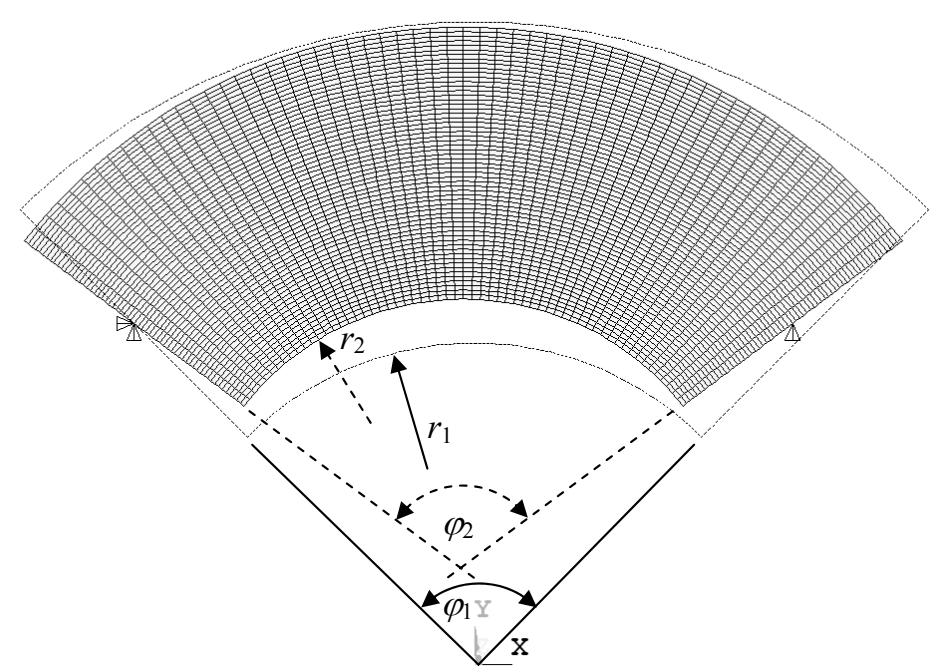

Bild 4 Verformungsfigur (Netz), unmaßstäblich, für das statisch bestimmt gelagerte Kreisringstück; $r_{1}$ und $r_{2}$ beispielhaft jeweils am inneren Radius dargestellt

Fig. 4 Deformed structure (mesh), not scaled, part of a circular ring statically determinate supported; $r_{1}$ and $r_{2}$ shown as an example at the inner radius

Für das freie Schwinden oder Quellen eines Kreisringstücks aus Brettschichtholz gelten unter idealen Bedingungen (polar-orthotropes Material und konstante Feuchteverhältnisse) folgende Beziehungen (vgl. auch Keylwerth 1944/1945 sowie LarsenLarsen und Riberholt 1983): Für den Radius $\left(r_{2}\right)$ einer beliebigen Faser nach dem Schwinden oder Quellen gilt Gleichung (4)(4):

$$
r_{2}=r_{1} \cdot\left(1+\alpha_{\mathrm{t} / \mathrm{r}} \cdot \Delta u\right)
$$

und für den Winkel zwischen den Hirnflächen ( $\varphi_{2}$, in rad) nach dem Schwinden oder Quellen Gleichung (5)(5):

$$
\varphi_{2}=\varphi_{1} \cdot \frac{1+\alpha_{\ell} \cdot \Delta u}{1+\alpha_{\mathrm{t} / \mathrm{r}} \cdot \Delta u} .
$$

In den Gleichungen (4)(4) und (5) $(5)$ sind $r_{1}$ der Radius einer beliebigen Faser und $\varphi_{1}$ (in rad) der Winkel zwischen den Hirnflächen jeweils vor dem Schwinden oder Quellen. Für Schwinden sind $\alpha_{\ell}$ bzw. $\alpha_{\mathrm{t} / \mathrm{r}}$ mit negativem und für Quellen mit 
positivem Vorzeichen einzusetzen. Das gilt natürlich nur dann, wenn mit Schwinden eine Abnahme und mit Quellen eine Zunahme der Maße verbunden ist. Grundsätzlich wurden auch schon axiale Kürzungen beim Quellen beobachtet (z.B. Koponen et al. 1989).

Die Modellbetrachtungen am statisch unbestimmt und statisch bestimmt gelagerten Kreisringstück bedeuten für ingenieurmäßige Fragestellungen: In Zwei-Gelenk-Bogenträgern entstehen infolge von Holzfeuchte-Änderungen Zwangsspannungen und in gekrümmten Bereichen statisch bestimmt gelagerter Träger Krümmungsänderungen. Diese können dann wiederum Zwangsspannungen nach sich ziehen, wenn sich die Bauteile nicht unbehindert krümmen können.

\section{Berechnungsbeispiele}

1. Beispiel: Die folgende theoretische Berechnung soll zeigen, welche zusätzliche Biegerand- bzw. Querzugspannung entsteht, wenn sich die Krümmungszunahme $(\Delta \kappa)$ infolge einer konstanten Holzfeuchte-Abnahme von $5 \%$, eines bei Bauteilen aus Brettschichtholz durchaus zu erwartenden Unterschieds (DIN 1052:2008), im gekrümmten Bereich des in Bild 5 Bild 5 halbseitig dargestellten Satteldachträgers nicht unbehindert einstellen kann.

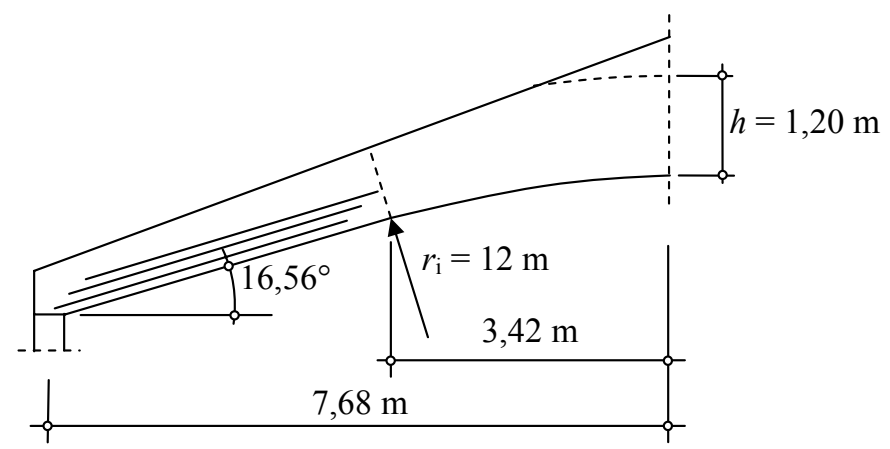

Bild 5 Beispielhaft gewählte Trägergeometrie zur Untersuchung der Auswirkungen der Anisotropie und Holzfeuchte-Änderungen auf die Verformung und Spannungszustände im gekrümmten Bereich

Fig. 5 Geometry of a pitched cambered beam used to study the effect of both differential shrinkage and moisture variations on the deformation and stresses in the curved zone

Für den Radius der Schwerelinie nach dem Schwinden folgt mit Gleichung (4)(4): 


$$
r_{2}=r_{1} \cdot\left(1+\alpha_{\mathrm{t} / \mathrm{r}} \cdot \Delta u\right)=(12+0,60) \cdot(1-0,0024 \cdot 5)=12,45 \mathrm{~m} .
$$

Dem entspricht eine Krümmungszunahme von:

$$
\Delta \kappa=\frac{1}{r_{2}}-\frac{1}{r_{1}}=\frac{1}{12,45}-\frac{1}{12,6}=0,964 \cdot 10^{-3} \frac{1}{\mathrm{~m}} .
$$

Wenn diese Zunahme voll behindert wird, entsteht ein Zwangsmoment $(\Delta M)$ in der Größenordnung (Elastizitätsmodul $(E)$ in Faserrichtung von GL24, Trägheitsmoment 2. Grades (I), Trägerbreite (b)):

$$
\Delta M=\Delta \kappa \cdot E I=0,964 \cdot 10^{-3} \cdot 11600 \cdot \frac{1,20^{3} \cdot b}{12}=1,61 \cdot b \quad \mathrm{MN} .
$$

Unter Vernachlässigung der nichtlinearen Spannungsverteilung in gekrümmten Trägern entspricht dem Zusatzmoment eine zusätzliche Biegespannung am unteren Rand $\left(\Delta \sigma_{\mathrm{m}}\right)$ von etwa:

$$
\Delta \sigma_{\mathrm{m}} \approx \frac{\Delta M}{W}=\frac{1,61 \cdot b \cdot 6}{1,20^{2} \cdot b}=6,71 \mathrm{~N} / \mathrm{mm}^{2} .
$$

Diese fällt vergleichsweise hoch aus, weil in der Berechnung

Systemnachgiebigkeiten, die z.B. durch Schub- und Längsverformungen bedingt sind, unberücksichtigt bleiben. Nach DIN 1052:2008 liegen die entsprechenden Querzugspannungen dann in einer Größenordnung von maximal:

$$
\sigma_{\mathrm{t}, 90}=0,25 \cdot \frac{h}{r_{1}} \cdot \frac{\Delta M}{W}=0,25 \cdot \frac{1,20}{12,6} \cdot 6,71=0,16 \mathrm{~N} / \mathrm{mm}^{2} .
$$

Die Verhältnisse bei wirklichen Tragsystemen sind allerdings weitaus komplizierter: Eine vollständige Krümmungsbehinderung, die bei der Modellbetrachtung angenommen wurde, ist unwahrscheinlich und schließlich müssen die Spannungszustände für den Lastfall Holzfeuchte-Änderung mit Zwangskräften im Gleichgewicht stehen. Es ist daher im Lastfall HolzfeuchteÄnderung mit schwer zu fassenden Spannungszuständen und ggf. unzuträglichen Querzugspannungen zu rechnen, wenn beispielsweise die horizontale Verschiebung der Auflager von Satteldachträgern in steif eingespannten Stahlbetonstützen oder von Zwei-Gelenk-Bogenbindern nur eingeschränkt möglich ist. 
Ein weiteres Problem ist denkbar, wenn z.B. bei kombinierten Trägern in den Rand- und Kernlamellen jeweils systematisch unterschiedliche Schwind- und Quellmaße in Faserrichtung vorliegen. Da der Aufbau kombinierter Brettschichtholzträger in den 1960er Jahren bereits nutzbringend eingesetzt wurde (Egner 1963) und in DIN 1052:1969 differenziert geregelt war, wäre es grundsätzlich möglich, dass die folgenden Überlegungen mit den in Tabelle 1 Tabelle 1 analysierten Schäden zusammenhängen.

PerstorperPerstorper et al. (2001) und KligerKliger et al. (2003) zeigten in ihren experimentellen Untersuchungen zum Verformungsverhalten von Kanthölzern infolge Holzfeuchte-Abnahme im hygroskopischen Bereich ( $7 \%<u<16 \%$ ), dass das Schwindma $\beta$ in Faserrichtung und die Rohdichte mit $r=-0,62$ korreliert sind. Außerdem berichten sie, dass das Schwindmaß in Faserrichtung mit zunehmendem Abstand von der Markröhre, mit abnehmender Jahrringbreite und mit abnehmender Ästigkeit kleiner wird. Für kombiniertes Brettschichtholz bedeutet das möglicherweise ein tendenziell höheres Schwindverhalten in Faserrichtung im Kern- als im Randbereich. Welche Folgen das für einen kombinierten Satteldachträger haben kann, soll das zweite Berechnungsbeispiel deutlich machen.

\section{Beispiel: Mit den Steifigkeitskennwerten der Festigkeitsklasse C24 für}

Randlamellen und C16 für Kernlamellen wurde der gekrümmte Bereich des in Bild 5Bild 5 dargestellten Satteldachträgers untersucht. Das Kreisringstück wurde in der Symmetrieachse statisch bestimmt gelagert (vgl. Bild 6Bild 6) und mit der Finite-Elemente-Methode berechnet. Es wurden polar-orthotropes Materialverhalten, für die Randlamellen (jeweils äußere $h / 6$ ) ein Schwind- und Quellmaß in Faserrichtung von 0,005 \%/\% und quer dazu von $0,25 \% / \%$ und für die Kernlamellen (innere 4h/6) Schwind- und Quellmaße von 0,01 \%/\% bzw. 0,30 $\% / \%$ festgelegt. Die im Rand- und Kernbereich voneinander unterschiedlichen Schwind- und Quellmaße in Faserrichtung, in Anlehnung an die von PerstorperPerstorper et al. (2001) ermittelten Werte festgelegt, könnten durchaus wirklichkeitsnahe Verhältnisse in kombiniertem Brettschichtholz widerspiegeln. Die Steifigkeitskennwerte der Elemente in der ersten radialen Spalte jeweils neben den Hirnflächen wurden so gewählt, dass Schubverformungen weitgehend blockiert sind. Somit wurde die Modellierung des Satteldachträgers auf den 
gekrümmten Bereich reduziert, was für diese grundlegenden Betrachtungen ausreichend ist. Die Verformung des gekrümmten Bereichs und die entsprechenden Spannungszustände wurden für eine im gekrümmten Bereich konstante Holzfeuchte-Abnahme bzw. Zunahme von $5 \%$ berechnet. Die Holzfeuchte im Ausgangszustand läge dabei jeweils bei 12\%. Der Lastfall Holzfeuchte-Änderung bewegt sich damit im hygroskopischen Bereich zwischen 7\% und $17 \%$. Zwischen Schwinden oder Quellen und Holzfeuchte-Änderungen gilt dann näherungsweise ein linearer Zusammenhang und die Schwindmaße von PerstorperPerstorper et al. (2001) sind in diesem Bereich wirksam. Es wird angenommen, dass ihre an skandinavischer Fichte ermittelten Schwindmaße auch allgemein für Fichte gültig seien.

In Bild 6Bild 6 ist halbseitig die verformte Struktur im Vergleich mit der Kontur der unverformten dargestellt. Die in der Grafik links oben angegebene maximale Verschiebung $(D M X)$ von $0,0157 \mathrm{~m}$ entspricht der absoluten Verschiebung der beiden Ecken am äußeren Kreisbogen.

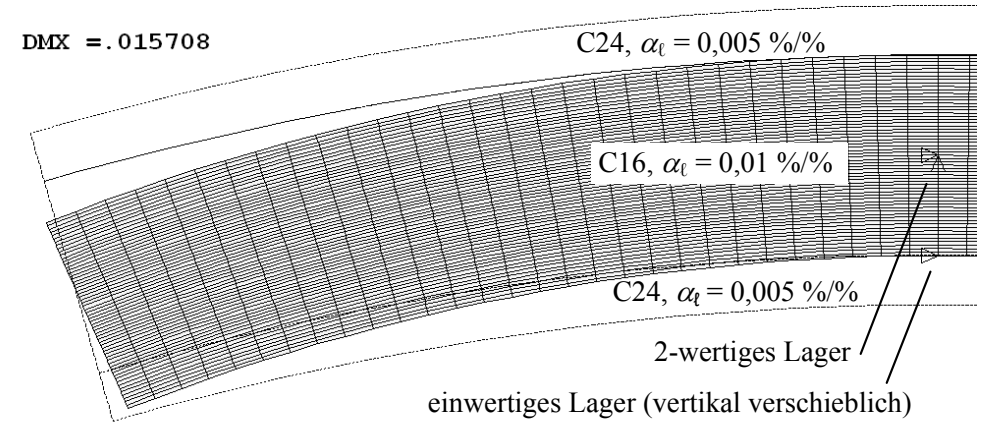

Bild 6 Unverformte (Linien) und verformte Struktur (Netz), unmaßstäblich, des gekrümmten Trägerbereichs; die beiden inneren Kreisbögen kennzeichnen die C24-C16-Klassengrenze bzw. die Grenze zwischen den unterschiedlichen Schwind- und Quellmaßen $\left(\alpha_{\ell}\right)$; maximale Verschiebung (mit $D M X$ bezeichnet) $=0,0157 \mathrm{~m}$

Fig. 6 Undeformed (lines) and deformed structure (mesh), not scaled, of the curved zone; the two inner lines indicate the dividing boundaries between the lamination grades C24 and $\mathrm{C} 16$ and between the shrinkage coefficients in longitudinal direction $\left(\alpha_{\ell}\right)$; maximum displacement (labelled as $D M X)=0.0157 \mathrm{~m}$ 
Die infolge Schwindens in der gekrümmten Ebene sich einstellenden

Eigenspannungen sind in Bild 7Bild 7 bis Bild 9Bild 9 dargestellt. Darin werden Spannungen in Faserrichtung mit $S Y$, Spannungen quer zur Faser mit $S X$ und Schubspannungen mit $S X Y$ gekennzeichnet. Die Einheit ist jeweils N/mm². Die drei Spannungen beziehen sich auf ein globales zylindrisches Koordinatensystem mit Ursprung im Krümmungsmittelpunkt des Kreisringstücks. $X$ kennzeichnet die globale radiale und $Y$ die globale tangentiale Richtung. Die Spannungszustände für $S Y$ und $S X$ lassen sich folgendermaßen erklären: Da bei jeweils gleicher Holzfeuchte-Abnahme die Randlamellen weniger schwinden als die Kernlamellen, bauen sich in den Randlamellen Druck- (Bild 7Bild 7, in Weiß) und in den Kernlamellen Zugspannungen (Bild 7Bild 7, in Dunkelgrau) auf.

Gekrümmte druckbeanspruchte Bauteile sind bestrebt sich weiter zu krümmen, wohingegen zugbeanspruchte gekrümmte Bauteile dazu neigen, eine gerade Form anzunehmen. Dieses unterschiedliche, voneinander getrennt betrachtetes Verformungsverhalten der Rand- und Kernlamellen steht im Einklang mit den Spannungen quer zur Faser (Bild 8Bild 8): Im oberen Drittel des Kreisringstücks herrschen Zugspannungen (dunkelgrau) und im unteren Drittel Druckspannungen (hellgrau). Die Ausprägung der Schubspannungen (Bild 9Bild 9), nur im gekrümmten Bereich, ist erwartungsgemäß: Sie sind vergleichsweise klein.

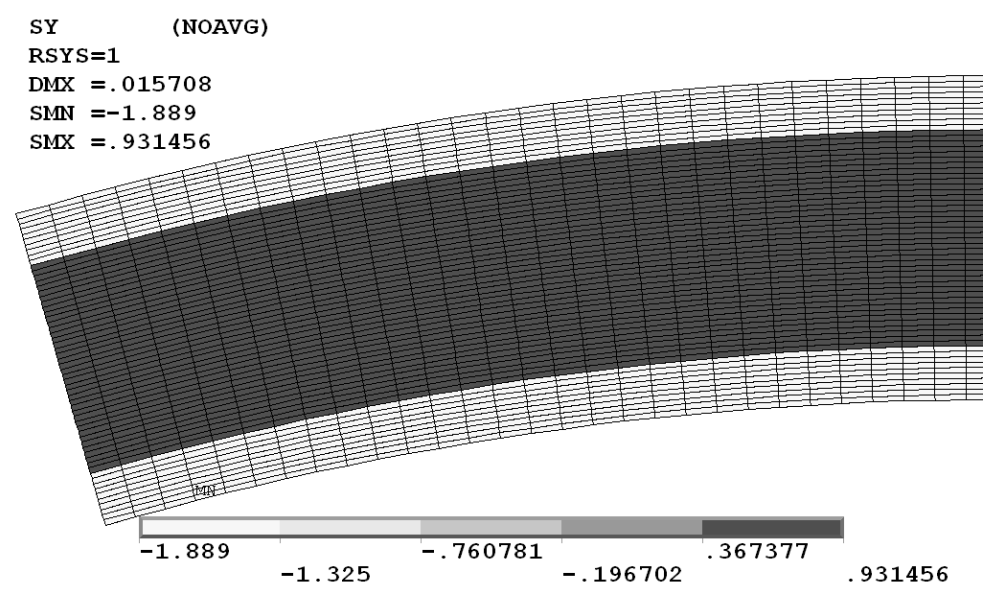

Bild 7 Spannungen in Faserrichtung $(S Y)$, bezogen auf das globale zylindrische

Koordinatensystem (durch $R S Y S=1$ angezeigt) mit Ursprung im Krümmungsmittelpunkt des Kreisringstücks und $X$ in radialer und $Y$ in tangentialer Richtung; keine Durchschnittswerte in der Grafik (durch $N O A V G$ angezeigt); Minimum $S Y(S M N)=-1,89$ $\mathrm{N} / \mathrm{mm}^{2}$; Maximum $S Y(S M X)=0,931 \mathrm{~N} / \mathrm{mm}^{2}$ 
Fig. 7 Stresses in grain direction $(S Y)$, related to the global cylindrical coordinate system (indicated by $R S Y S=1$ ) with origin in the centre of curvature of the circular ring and $X$ in radial $Y$ in tangential direction; unaveraged values in the contour plot (indicated by $N O A V G$ ); minimum $S Y(S M N)=-1.89 \mathrm{~N} / \mathrm{mm}^{2} ;$ maximum $S Y(S M X)=0.931 \mathrm{~N} / \mathrm{mm}^{2}$

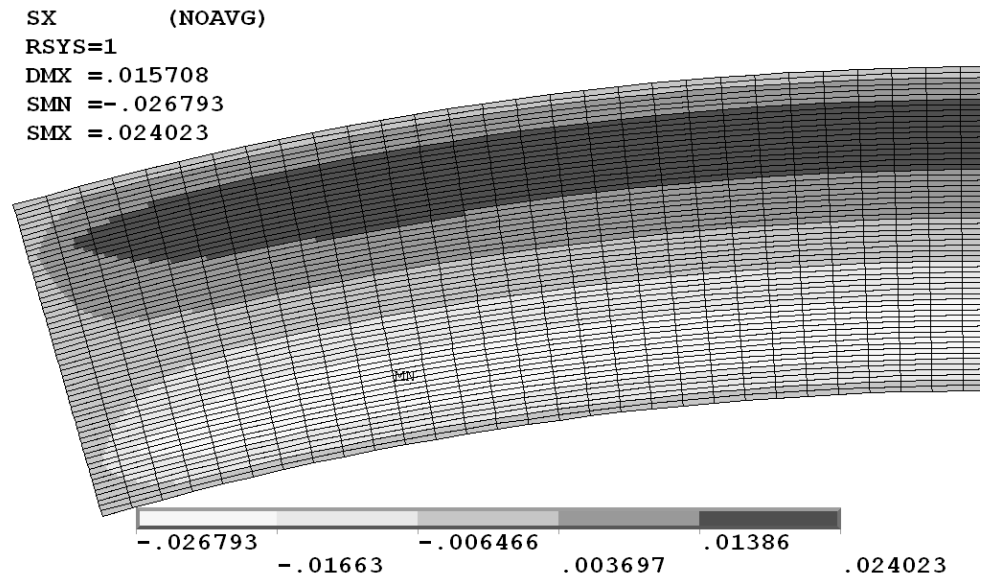

Bild 8 Spannungen quer zur Faserrichtung $(S X)$, bezogen auf das globale zylindrische Koordinatensystem (s. Bildunterschrift 7); Minimum $S X(S M N)=-0,0268 \mathrm{~N} / \mathrm{mm}^{2}$; Maximum $S X(S M X)=0,0240 \mathrm{~N} / \mathrm{mm}^{2}$

Fig. 8 Stresses perpendicular to the grain $(S X)$, related to the global cylindrical coordinate system (see caption of Figure 7); minimum $S X(S M N)=-0.0268 \mathrm{~N} / \mathrm{mm}^{2}$; maximum $S X$ $(S M X)=0.0240 \mathrm{~N} / \mathrm{mm}^{2}$

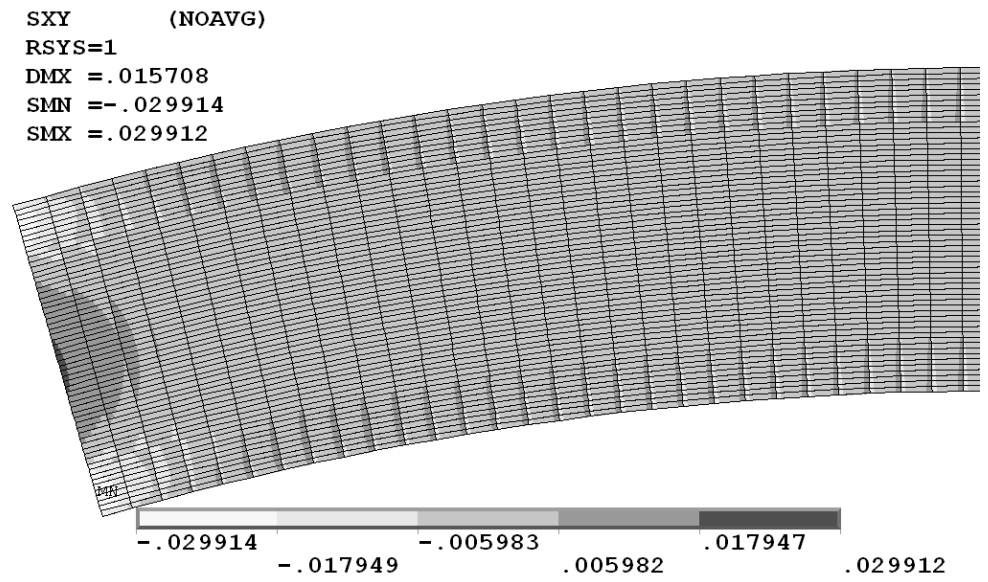


Bild 9 Schubspannungen $(S X Y)$, bezogen auf das globale zylindrische Koordinatensystem (s. Bildunterschrift 7); Minimum $S X Y(S M N)=-0,0299 \mathrm{~N} / \mathrm{mm}^{2}$; Maximum $S X Y(S M X)=$ $0,0299 \mathrm{~N} / \mathrm{mm}^{2}$

Fig. 9 Shear stresses $(S X Y)$, related to the global cylindrical coordinate system (see caption of . Figure 7); minimum $S X Y(S M N)=-0.0299 \mathrm{~N} / \mathrm{mm}^{2} ;$ maximum $S X Y(S M X)=0.0299$ $\mathrm{N} / \mathrm{mm}^{2}$

Anhand der Spannungswerte in Bild 8Bild \& kann als maximale Querzugspannung $\left(\sigma_{\mathrm{t}, 90, \max }\right)$ der Wert $0,024 \mathrm{~N} / \mathrm{mm}^{2}$ festgelegt werden. Mit dem

Teilsicherheitsbeiwert $\left(\gamma_{\mathrm{F}}\right)$ von 1,5 für Einwirkungen aus Zwang und dem Modifikationsbeiwert $\left(k_{\mathrm{mod}}\right)$ von 0,6 für ständige Einwirkungen folgt:

$$
\begin{aligned}
& \sigma_{\mathrm{t}, 90, \mathrm{~d}}=\gamma_{\mathrm{F}} \cdot \sigma_{\mathrm{t}, 90, \max } \approx 1,5 \cdot 0,024=0,036 \mathrm{~N} / \mathrm{mm}^{2} \\
& f_{\mathrm{t}, 90, \mathrm{~d}} \approx \frac{k_{\mathrm{mod}} \cdot f_{\mathrm{t}, 90, \mathrm{k}}}{\gamma_{\mathrm{M}}}=\frac{0,6 \cdot 0,5}{1,3}=0,23 \mathrm{~N} / \mathrm{mm}^{2} \\
& \frac{\sigma_{\mathrm{t}, 90, \mathrm{~d}}}{f_{\mathrm{t}, 90, \mathrm{~d}}} \cdot 100=16 \%
\end{aligned}
$$

Die Berechnung (6) $(6)$ des Beispiels zeigt, dass der Lastfall Schwinden mit $\Delta u=-5 \%$ zu einem sich räumlich weit erstreckenden Bemessungswert der Querzugspannung $\left(\sigma_{\mathrm{t}, 90, \mathrm{~d}}\right)$ führt, der etwa $1 / 6$ der Festigkeit $\left(f_{\mathrm{t}, 90, \mathrm{~d}}\right)$ beträgt und daher nicht mehr vernachlässigt werden sollte.

Beim Lastfall Quellen mit betragsmäßig gleicher Holzfeuchte-Änderung wie beim Schwinden kehren sich in Bild 7Bild 7 bis Bild 9Bild 9 lediglich die Vorzeichen der Spannungen um. Der Lastfall Quellen ist damit ebenso kritisch wie der Lastfall Schwinden. Numerische Ergebnisse hinsichtlich des Quellens und die Querzugproblematik auch bei Satteldachträgern, die Holzfeuchten von deutlich über $12 \%$ aufweisen (Blaß und Frese 2010), würden sich also nicht widersprechen.

\section{Zusammenfassung}

Mit den Erkenntnissen über das anisotrope Schwinden und Quellen in der Hirnfläche von Holz, mit einfachen Modellbetrachtungen sowie mit Beispielen von einem analytisch und einem numerisch berechneten Kreisringstück aus Brettschichtholz wurden ingenieurmäßige Überlegungen zur Wechselwirkung zwischen der Anisotropie der Schwind- und Quellmaße in der Ebene von 
gekrümmtem Brettschichtholz und Holzfeuchte-Änderungen angestellt. Es konnte gezeigt werden, dass die verhältnismäßig stark ausgeprägte Anisotropie und nutzungsbedingte Holzfeuchte-Änderungen eine Zu- oder Abnahme der Bauteilkrümmung gemeinsam bedingen. Die Anisotropie bei Brettschichtholz ist dabei durch ein Verhältnis aus Schwind- und Quellmaß in und quer zur Faserrichtung von etwa 1/24 gekennzeichnet. Krümmungsänderungen ziehen bei statisch unbestimmten Systemen Zwangsspannungen nach sich; bei statisch bestimmten Systemen können sich bei ungewollt behinderten Auflagerverschiebungen Zwangsspannungen einstellen. Diese liegen fallweise in einer Größenordnung, die nicht mehr vernachlässigt werden kann. Grundsätzlich ist es sogar denkbar, dass bei kombiniertem Brettschichtholz aufgrund von Schwind- und Quellmaßen in Faserrichtung, die nach wissenschaftlichen Erkenntnissen bei den Randlamellen systematisch kleiner sein können als bei den Kernlamellen, infolge Holzfeuchte-Änderungen Eigenspannungen entstehen. Bei gekrümmten kombiniert aufgebauten Bauteilen treten dann u. a. in weiten Bereichen Querzugspannungen auf.

Es ist möglich, dass hier aufgezeigte Effekte für die bis heute ausgeprägte Querzugproblematik bei Satteldachträgern aus Brettschichtholz mit ursächlich sind. Für genauere Kenntnisse von den wahren Spannungszuständen sind noch gezielte numerische Untersuchungen notwendig. Einige offene Fragen betreffen den Einfluss der Modellbildung (z.B. Feuchtegefälle in der Brettschichtholzebene beim Schwinden oder Quellen), von zufällig streuenden und von stark streuenden Schwind- und Quellmaßen (z.B. Brettchargen mit Druckholz), von unterschiedlichen Trägerformen (z.B. gerade kombinierte Träger, kombinierte Fischbauchträger) und den Einfluss der Relaxation jeweils auf die Spannungszustände und auf die Ausprägung der Spannungen. Gegebenfalls sind experimentelle Untersuchungen an gekrümmten Bauteilen erforderlich.

\section{Literatur}

Blaß HJ, Frese M (2010) Schadensanalyse von Hallentragwerken aus Holz. Forschungsbericht der Versuchsanstalt für Stahl, Holz und Steine, Abt. Ingenieurholzbau, Universität Karlsruhe (TH). Noch unveröffentlicht Egner K (1963) Leimbauweisen. In: v. Halász R (Hg.): Holzbau Taschenbuch. 6. Aufl., Wilhelm Ernst \& Sohn, Berlin, München 
Hoffmeyer P (1995) Holz als Baustoff Step 1. Fachverlag Holz der Arbeitsgemeinschaft Holz e.V., Düsseldorf

Hsu NN, Tang RC (1975) Distortion and Internal Stresses in Lumber Due to Anisotropic Shrinkage. Wood Science 7: 298-307

Kang W, Lee NH (2002) Mathematical modeling to predict drying deformation and stress due to the differential shrinkage within a tree disk. Wood Sci Technol 36: 463-476

Kang W, Lee NH (2004) Relationship between radial variations in shrinkage and drying defects of tree disks. Journal of Wood Science 50: 209-216

Keylwerth R (1944/1945) Das Schwinden und seine Beziehungen zu Rohwichte und Aufbau des Holzes. Holz Roh- Werkst 7: 7-21

Keylwerth R (1951) Formänderungen in Holzquerschnitten. Holz Roh- Werkst 9: 253-260

Keylwerth R (1962) Untersuchungen über freie und behinderte Quellung von Holz - Erste Mitteilung: Freie Quellung. Holz Roh- Werkst 20: 252-259

Kliger R, Johansson M, Perstorper M, Johansson G (2003) Distortion of Norway spruce timber; Part 3. Modelling bow and spring. Holz Roh- Werkst 61: 241-250

Kollmann F (1982) Technologie des Holzes und der Holzwerkstoffe. 2. Aufl., Springer-Verlag, Berlin, Göttingen, Heidelberg

Koponen S, Toratti T, Kanerva P (1989) Modelling longitudinal elastic and shrinkage properties of wood. Wood Sci Technol 23: 55-63

Kubler H (1975) Study on Drying of Tree Cross Sections. Wood Science 7: 173-181

Larsen HJ, Riberholt H (1983) Trækonstruktioner, Beregning. SBI-Anvisning 135, Statens Byggeforskningsinstitut, Denmark

Möhler K, Hemmer K (1981) Eingeleimte Gewindestangen. In: Arbeitsgemeinschaft Holz e. V. Düsseldorf (Hg.): Holzbau-Statik-Aktuell, Folge 6, Informationen zur Berechnung von

Holzkonstruktionen

Möhler K, Steck G (1980) Untersuchungen über die Rissbildung in Brettschichtholz infolge Klimabeanspruchung. Bauen mit Holz 4/1980: 194-200

Perstorper M, Johansson M, Kliger R, Johansson G (2001) Distortion of Norway spruce timber; Part 1. Variation of relevant wood properties. Holz Roh- Werkst 59: 94-103

Tauchert TR, Hsu NN (1977) Shrinkage Stresses in Wood Logs Considered as Layered, Cylindrically Orthotropic Materials. Wood Science and Technology 11: 51-58

DIN 1052:1969-10 Holzbauwerke - Berechnung und Ausführung

DIN 1052:1988-04 Holzbauwerke - Berechnung und Ausführung

DIN 1052:2004-08 Entwurf, Berechnung und Bemessung von Holzbauwerken - Allgemeine

Bemessungsregeln und Bemessungsregeln für den Hochbau 
Ejwwp443_source

DIN 1052:2008-12 Entwurf, Berechnung und Bemessung von Holzbauwerken - Allgemeine

Bemessungsregeln und Bemessungsregeln für den Hochbau

DIN 52184:1979-05 Prüfung von Holz - Bestimmung der Quellung und Schwindung

\section{Hilfsmittel}

Die Finite-Elemente-Berechnungen wurden mit dem Programmsystem ANSYS (Version 11.0) der ANSYS, Inc., Canonsburg, PA, USA durchgeführt. 